\title{
Production Systems and Contribution on Characterization of Local Chickens in Smallholder Farmer in Sud-Kivu Province, Democratic Republic of the Congo (DRC)
}

\author{
M. M. D. Katunga ${ }^{1,2^{*}}$, K. F. Balemirwe ${ }^{2 *}$, F. Masheka1 ${ }^{*}$, P. Zamukulu ${ }^{3 *}$ \\ ${ }^{1}$ INERA Mulungu, Bukavu, DRC \\ ${ }^{2}$ Faculté de médecine vétérinaire, Université du cinquantenaire Lwiro, DRC \\ ${ }^{3}$ Université Évangélique en Afrique (UEA), Faculté des Sciences Agronomiques et Environnement, \\ Bukavu-RDC, Cyangugu-Rwanda \\ Email: stylonya@gmail.com, katungamusale@yahoo.fr, katungamusale@inera-rdc.org, fidelkatunga@yahoo.fr, \\ bmasheka@yahoo.fr
}

How to cite this paper: Katunga, M.M.D., Balemirwe, K.F., Masheka, F. and Zamukulu, P. (2020) Production Systems and Contribution on Characterization of Local Chickens in Smallholder Farmer in Sud-Kivu Province, DRC. Open Access Library Journal, 7: e6171.

https://doi.org/10.4236/oalib.1106171

Received: February 17, 2020

Accepted: March 17, 2020

Published: March 20, 2020

Copyright (C) 2020 by author(s) and Open Access Library Inc.

This work is licensed under the Creative Commons Attribution International License (CC BY 4.0).

http://creativecommons.org/licenses/by/4.0/

(c) (i) Open Access

\begin{abstract}
Sud-Kivu province in DRC faces to malnutrition of his population. Agriculture and especially livestock have a low yield due to bad government, low diseases control, lack of feed, inappropriate chicken's accommodation. Importation of food is very high. A survey was implemented on local chickens rearing in Bukavu town and his hinterland territories of Kabare, Walungu and Kalehe in Sud-Kivu. Conducted at the household level, standard methods of interviews and structured questionnaires were used on characterization chicken's production systems and commercialization. Survey began on $2^{\text {nd }}$ and finished on $22^{\text {th }}$ April 2016. Results showed in terms of hatching, weaning, laying, health, accommodation, eggs production, weights and prices of eggs, hen and coq that local chickens still were mostly reared in traditional systems. Chicken's products prices were high. To start a good program of chicken industry in this province and as well as in entire the country, selection of local chickens should before be carried out on characterization of production systems and genetic molecular analysis.
\end{abstract}

\section{Subject Areas}

Zoology

\section{Keywords}

RDC, Survey, Chickens, Production Systems, Market 


\section{Introduction}

Livestock production in Sud-Kivu DRC decreased drastically due to looting during the various wars ended in 1999, even if now, some troubles persist in eastern part of the country. The diminution of livestock is also due to the consequence of low extension services [1], lack of animal feed, bad governance, lack of farmer's support, low diseases control etc. Despite these bad performances, livestock still been managed on extensive system [2]. In Sud-Kivu, consumption of red meat, pork and chicken was low in all Mandate Areas with an exception of Ruzizi plains where the average consumption frequency of red meat was 4 times a month [3]. Meanwhile, Sud-Kivu and all the country have a huge agricultural potential characterized by sufficient rainfall, a major river system, a high diversity of soils and broad sunshine [4]. The rate of malnutrition among the population is very high $15 \%$ [5]. To fight hunger and proteins malnutrition, monogastric animals like chickens can help to produce quickly quality meat and eggs. In this way, chickens shall be encouraged to fight against malnutrition and poverty. The aim of this study was to contribute on local chicken characterization production systems based on the KAFACI project on the promotion of good management for increased productivity of market oriented small scale chicken producers in DRC.

\section{Material and Methods}

\subsection{Region Surveyed}

Sud-Kivu province covers $69,130 \mathrm{~km}^{2}$ and population is estimated at $6,432,984$ people. This province is located in eastern part of DRC between $1^{\circ} 36^{\prime}$ of latitude South and $5^{\circ}$ of latitude South at one hand and $26^{\circ} 47^{\prime}$ of longitude East and $29^{\circ} 20^{\prime}$ and longitude East at the other hand [6]. Survey was implemented in $\mathrm{Bu}$ kavu principal town and his hinterland of around $50 \mathrm{~km}$, see Figure 1 in the territories of Kabare, Walungu and Kalehe.

\subsection{Survey Method Applied}

Interview was carried out on $2^{\text {nd }}$ to $22^{\text {th }}$ April 2015 using standard methods of interviews and structured questionnaires at the household level. Site sampling was randomly done. Questionnaire was submitted to 18 sites; in Bukavu town and his hinterland for at all 180 stakeholders with 10 households per street and village.

\subsection{Data Analysis}

Data were collected on the socio demographic characteristics, on production practices adopted by farmers, stock size and composition, management and housing systems adopted, feeding systems, diseases, weights and prices taken on chicken products. Then, households were interviewed in Bukavu town in 9 streets and in his hinterland 9 villages. The number of animals was converted 


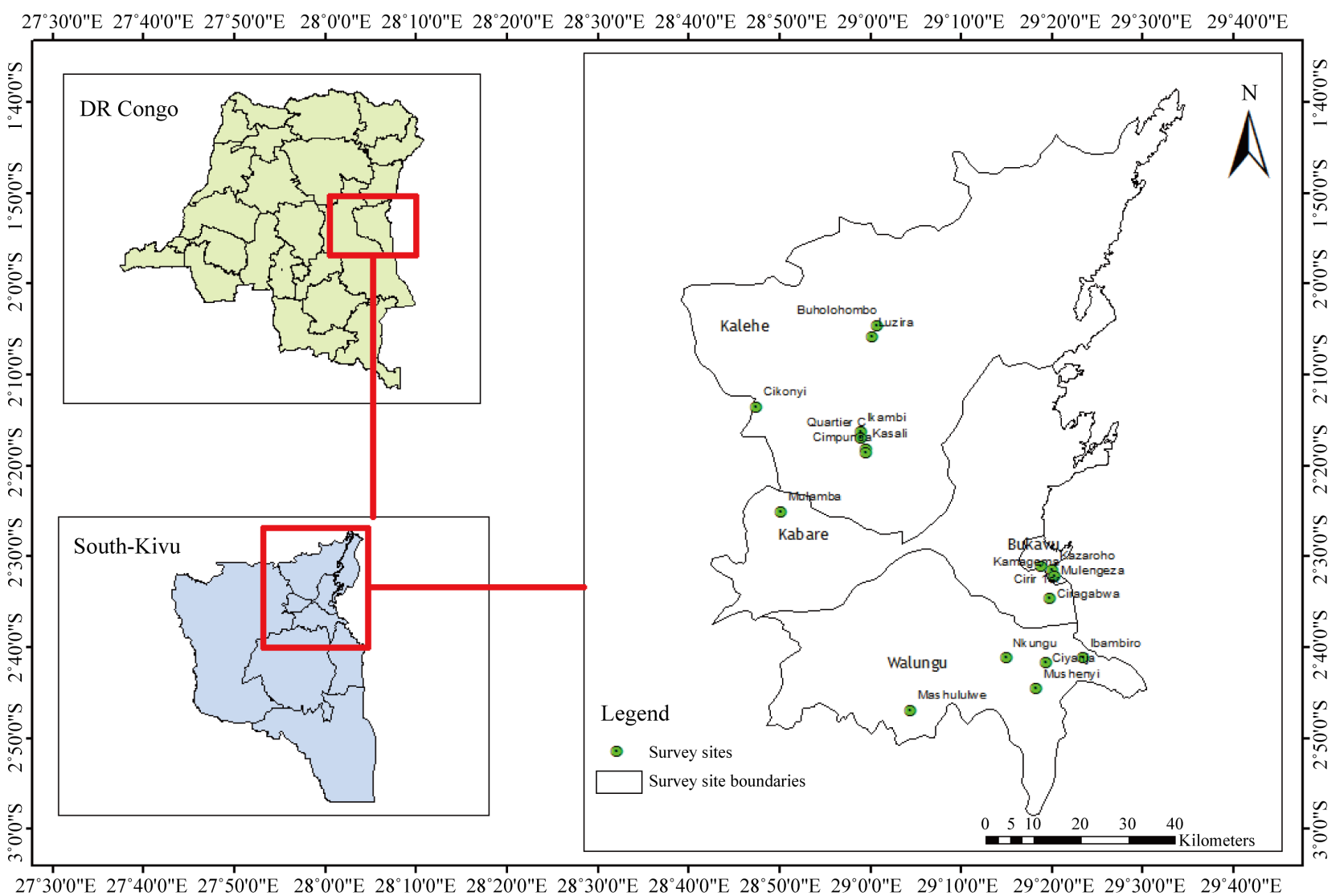

Figure 1. Sites localisation of survey in Sud-Kivu.

into Tropical Livestock Unit (TLU), where cattle are weighed with 0.7, sheep/goat 0.1, swine with 0.2 and chicken 0.01 [7], duck was assigned 0.02 TLU, rabbit 0.01 TLU, cavy 0.005 TLU [8]. Materials used were GPS and an electronic scale "Electronic Kitchen scale SF-400" to weight eggs and chickens. Data were analyzed using descriptive analysis such as frequency distribution, percentages and means comparison on IBM SPSS Statistics version 20 software.

\section{Results}

\subsection{Social Farmer's Characterization}

Regarding on Table 1, gender issue represented male at $66.3 \%$ and female $36.7 \%$. Gender balance is observed in six sites, eleven had at least $40 \%$ of females and one had $60 \%$ of females. The average age of the farmers was $39.3 \pm 14.5$ years, although there were significant $(\mathrm{P}<0.05)$ differences in the average age of the farmers across the sites. Mushenyi site had the oldest farmers with an average of $49.3 \pm 9.5$ years when the youngest was in Kasali site with $23.6 \pm 11.4$ years. The education level indicted that majority of farmers did the secondary school $41.7 \%$ followed by primary school 31.7 , illiterate $22.2 \%$ and university $4.4 \%$. The chicken's experience from grouping age demonstrated that 0 up to 5 years the average of years' experience in chicken production was $2.6 \pm 1.5$ years, 
Table 1. Socio economic farmer's characterization.

\begin{tabular}{|c|c|c|c|c|c|c|c|c|c|c|}
\hline \multirow{2}{*}{$\begin{array}{l}\text { Streets and } \\
\text { villages }\end{array}$} & \multicolumn{2}{|c|}{ Gender } & \multirow[t]{2}{*}{ Farmer's age } & \multicolumn{4}{|c|}{ Education } & \multicolumn{3}{|c|}{ Years experience } \\
\hline & Male & Female & & Illiterate & Primary & Secondary & University & Up to 5 & $6-10$ & Above 10 \\
\hline Buholohombo & 7 & 3 & $42.6 \pm 12.7$ & 1 & 2 & 7 & & 1.5 & $7.5 \pm 0.7$ & $28.4 \pm 9.7$ \\
\hline Cikonyi & 6 & 4 & $43.4 \pm 13.0$ & 2 & 5 & 3 & & $3.0 \pm 1.7$ & $7.0 \pm 1.4$ & $29.0 \pm 10.2$ \\
\hline Cimpunda & 5 & 5 & $33.5 \pm 13.7$ & & 2 & 6 & 2 & $1.8 \pm 0.9$ & 6.0 & 30.0 \\
\hline Cirhagabwa & 5 & 5 & $42.9 \pm 16.8$ & 4 & 5 & & 1 & $2.3 \pm 1.9$ & 10.0 & $20.0 \pm 6.1$ \\
\hline Ciriri & 6 & 4 & $35.9 \pm 14.4$ & 2 & 8 & & & $2.9 \pm 2.0$ & $8.7 \pm 2.3$ & \\
\hline Ciyanja & 8 & 2 & $43.1 \pm 16.3$ & 5 & 2 & 3 & & & 8.0 & $26.0 \pm 14.0$ \\
\hline Ibambiro & 5 & 5 & $39.3 \pm 21.4$ & 4 & 3 & 3 & & $3.7 \pm 1.5$ & $8.0 \pm 2.8$ & $26.2 \pm 21.2$ \\
\hline Ikambi & 6 & 4 & $30.0 \pm 12.9$ & 2 & 1 & 7 & & $3.0 \pm 1.2$ & 7.0 & $26.4 \pm 11.3$ \\
\hline Kamagema & 5 & 5 & $38.1 \pm 12.1$ & 1 & 1 & 7 & 1 & 1.0 & $7.0 \pm 1.4$ & $25.3 \pm 8.6$ \\
\hline Kasali & 9 & 1 & $23.6 \pm 11.4$ & & 4 & 5 & 1 & $1.6 \pm 0.9$ & $7.5 \pm 2.1$ & $19.0 \pm 1.4$ \\
\hline Kazaroho & 4 & 6 & $30.2 \pm 9.2$ & 1 & 2 & 7 & & $2.2 \pm 1.6$ & $7.3 \pm 2.3$ & 30.0 \\
\hline Luzira & 5 & 5 & $39.5 \pm 11.9$ & 3 & 2 & 4 & 1 & $3.3 \pm 0.6$ & & $19.1 \pm 5.6$ \\
\hline Mashululwe & 6 & 4 & $34.3 \pm 12.2$ & 2 & 6 & 2 & & $2.3 \pm 2.3$ & 8.0 & $18.5 \pm 6.7$ \\
\hline Mulamba & 5 & 5 & $45.8 \pm 17.2$ & 2 & 5 & 3 & & $3.5 \pm 2.1$ & & $27.5 \pm 12.5$ \\
\hline Mulengeza & 8 & 2 & $43.0 \pm 9.6$ & 2 & 2 & 5 & 1 & $3.7 \pm 0.6$ & 10.0 & $21.3 \pm 7.6$ \\
\hline Mushenyi & 9 & 1 & $49.3 \pm 9.5$ & 3 & 3 & 3 & 1 & & & $29.3 \pm 9.8$ \\
\hline Nkungu & 9 & 1 & $47.2 \pm 15.8$ & 4 & & 6 & & 5.0 & 10.0 & $35.9 \pm 11.6$ \\
\hline Quartier C & 6 & 4 & $38.1 \pm 12.7$ & 2 & 4 & 4 & & $3.3 \pm 1.7$ & $9.5 \pm 0.7$ & \\
\hline Total & $114(66.3)$ & $66(36.7)$ & $39.3 \pm 14.5$ & $40(22.2)$ & $57(31.7)$ & 75 (41.7) & $8(4.4)$ & $2.6 \pm 1.5$ & $8.1 \pm 1.7$ & $15.0 \pm 13.5$ \\
\hline $\begin{array}{c}\text { Test Stat. Value } \\
\text { (F) }\end{array}$ & & & $2.086^{*}$ & & & & & & & \\
\hline
\end{tabular}

${ }^{*}$ Significant at $\mathrm{P}<0.05$, values in parentheses are percentages.

6 to 10 years $8.1 \pm 1.7$ years and above 10 years, $15.0 \pm 13.5$ years.

\subsection{Livestock Production}

Various animals are reared in the study area, see Table 2. According to the TLU, the highest mean is recorded to swine $2.3 \pm 2.0 \mathrm{TLU}$, followed by cattle $2.2 \pm 2.9$ TLU, sheep $0.32 \pm 0.3$ TLU, goat $0.31 \pm 0.2$ TLU and chicken $0.26 \pm 1.7$ TLU were both significant $(\mathrm{P}<0.05)$ differences in TLU average across the sites, duck $0.12 \pm 0.5$ TLU, cavy $0.09 \pm 1.5$ TLU and Rabbit 0.04 TLU. Swine, goats and chickens were more reared in the region than the other animal species.

\subsection{Animals species Reared}

Table 2 shows the animal species. 
Table 2. Animals species (TLU).

\begin{tabular}{|c|c|c|c|c|c|c|c|c|}
\hline Sites & Swine & Cattle & Sheep & Goat & Duck & Chickens & Cavy & Rabbit \\
\hline Buholohombo & 12.0 & & & $0.40 \pm 0.1$ & & $0.08 \pm 0.1$ & 0.05 & \\
\hline Cikonyi & 1.0 & 2.1 & & $0.32 \pm 0.1$ & & $0.19 \pm 0.3$ & 0.01 & \\
\hline Cimpunda & & & & 0.30 & & $0.09 \pm 0.6$ & 0.05 & \\
\hline Cirhagabwa & $2.3 \pm 1.8$ & & & $0.17 \pm 0.1$ & & $0.11 \pm 0.1$ & 0.01 & \\
\hline Ciriri & $1.3 \pm 0.7$ & $1.17 \pm 0.8$ & $015 \pm 0.1$ & $0.20 \pm 0.1$ & & 0.14 & $0.05 \pm 0.5$ & 0.09 \\
\hline Ciyanja & $1.6 \pm 0.5$ & & & $0.38 \pm 0.2$ & 0.06 & $0.58 \pm 0.4$ & $0.21 \pm 0.3$ & 0.03 \\
\hline Ibambiro & $4.5 \pm 0.7$ & & & $0.27 \pm 0.1$ & & 0.05 & $0.04 \pm 0.2$ & $0.28 \pm 0.2$ \\
\hline Ikambi & 1.01 & $1.05 \pm 0.5$ & & $0.36 \pm 0.1$ & $0.13 \pm 0.6$ & $0.20 \pm 0.2$ & 0.08 & 0.06 \\
\hline Kamagema & $2.0 \pm 1.0$ & & 0.40 & $0.28 \pm 1.6$ & & $0.75 \pm 0.4$ & & 0.15 \\
\hline Kasali & 2.0 & & & 0.40 & & 0.07 & 0.03 & \\
\hline Kazaroho & $2.1 \pm 2.0$ & & & $0.18 \pm 0.1$ & & $0.11 \pm 0.7$ & & 0.37 \\
\hline Luzira & $2.4 \pm 2.6$ & $7.35 \pm 9.4$ & 1.0 & $0.48 \pm 0.3$ & & $0.09 \pm 0.1$ & 0.05 & 0.01 \\
\hline Mashululwe & $2.1 \pm 2.0$ & $0.93 \pm 0.4$ & 0.2 & $0.28 \pm 0.2$ & & 0.06 & $0.21 \pm 0.3$ & 0.15 \\
\hline Mulamba & $2.8 \pm 2.2$ & $3.68 \pm 2.3$ & & $0.30 \pm 0.1$ & & $0.07 \pm 0.5$ & 0.05 & $0.04 \pm 0.2$ \\
\hline Mulengeza & & & & $0.20 \pm 0.1$ & 0.07 & $0.05 \pm 0.3$ & 0.12 & 0.05 \\
\hline Mushenyi & $4.0 \pm 1.0$ & $1.47 \pm 1.2$ & & $0.48 \pm 0.2$ & & $0.09 \pm 0.1$ & $0.05 \pm 0.4$ & $0.63 \pm 0.4$ \\
\hline Nkungu & $1.7 \pm 0.8$ & $1.58 \pm 0.9$ & 0.2 & $0.25 \pm 0.1$ & & $0.09 \pm 0.6$ & $0.15 \pm 1.7$ & 0.04 \\
\hline Quartier C & 1.0 & 0.7 & & $0.40 \pm 0.4$ & & $0.11 \pm 0.7$ & 0.10 & 0.12 \\
\hline Total & $2.3 \pm 2.0$ & $2.22 \pm 2.9$ & $0.32 \pm 0.3$ & $0.31 \pm 0.2$ & $0.11 \pm 0.6$ & $0.09 \pm 0.1$ & $0.09 \pm 1.5$ & 0.04 \\
\hline Test Stat. Value (F) & & & & $1.819^{*}$ & & $1.881^{*}$ & & \\
\hline
\end{tabular}

${ }^{*}$ Significant at $\mathrm{P}<0.05$.

\subsection{Chicken Husbandry, Feed, Health}

Regarding Table 3, the average of clutches per year was $2.8 \pm 0.5$. Duration of clutch was $18.8 \pm 6.1$ days, eggs number per clutch was $14.7 \pm 4.2$. For incubation, the average of eggs number incubated per hen was $8.8 \pm 2.3$, number of eggs hatched $7.9 \pm 2.3$. The weaning period was $88.2 \pm 21.7$ days where chick's number at the weaning period was $5.2 \pm 2.8$. The rate of mortality was $34.1 \% \pm$ 25.2\%. Across the sites and when we consider the different parameters analyzed, there were high significant $(\mathrm{P}<0.001)$ differences in the average. Ciyanja site had a low rate of mortality $5.6 \% \pm 2.4 \%$ and Buholombo the highest with $68.7 \%$ $\pm 9.7 \%$.

At Table 4, hen usually laid egg once each day (61.1\%) and the mortality incidence per season was $68.5 \%$ in wet season.

Table 5 shows that the averages of chicken products weight were respectively $42.3 \pm 5.1 \mathrm{~g}$ of egg, $442.4 \pm 205.3 \mathrm{~g}$ of chicks at weaning pediod, $1265.5 \pm 282.1 \mathrm{~g}$ of hen and $1571.4 \pm 483.0 \mathrm{~g}$ of cock. The weight performances within the sites were performed for egg in Mushenyi $46.6 \pm 5.2 \mathrm{~g}$, chicks at weaning period in 
Table 3. Parameters carried out on local chicken's production (averages).

\begin{tabular}{|c|c|c|c|c|c|c|c|c|}
\hline Sites & $\begin{array}{l}\text { Clutch } \\
\text { number } \\
\text { per year }\end{array}$ & $\begin{array}{l}\text { Duration of } \\
\text { clutch } \\
\text { (days) }\end{array}$ & $\begin{array}{l}\text { Eggs number } \\
\text { per clutch }\end{array}$ & $\begin{array}{l}\text { Eggs number } \\
\text { incubated } \\
\text { per hen }\end{array}$ & $\begin{array}{c}\text { Eggs number } \\
\text { hatched } \\
\text { per hen }\end{array}$ & $\begin{array}{l}\text { Weaning } \\
\text { time } \\
\text { (days) }\end{array}$ & $\begin{array}{l}\text { Chicks number } \\
\text { at weaning } \\
\text { period }\end{array}$ & $\begin{array}{c}\text { Mortality } \\
\text { rate }\end{array}$ \\
\hline Buholohombo & $2.8 \pm 0.4$ & $23.2 \pm 7.4$ & $15.1 \pm 3.1$ & $9.5 \pm 1.6$ & $8.5 \pm 1.6$ & $81.0 \pm 21.4$ & $5.2 \pm 3.0$ & $68.7 \pm 9.7$ \\
\hline Cikonyi & $3.3 \pm 0.5$ & $25.4 \pm 6.7$ & $19.4 \pm 1.9$ & $11.9 \pm 2.3$ & $10.5 \pm 1.4$ & $87.0 \pm 17.0$ & $9.6 \pm 3.6$ & $25.8 \pm 10.8$ \\
\hline Cimpunda & $2.9 \pm 0.6$ & $18.7 \pm 3.7$ & $12.9 \pm 3.5$ & $7.9 \pm 2.6$ & $6.7 \pm 2.1$ & $57.0 \pm 11.8$ & $4.8 \pm 2.3$ & $28.2 \pm 17.2$ \\
\hline Cirhagabwa & $3.0 \pm 0.0$ & $23.4 \pm 8.0$ & $15.5 \pm 4.6$ & $8.4 \pm 1.3$ & $7.8 \pm 1.8$ & $78.0 \pm 25.3$ & $5.9 \pm 2.3$ & $33.9 \pm 20.4$ \\
\hline Ciriri & $3.0 \pm 0.0$ & $15.5 \pm 4.1$ & $15.5 \pm 3.9$ & $9.8 \pm 1.2$ & $9.4 \pm 1.5$ & $83.3 \pm 20.0$ & $6.0 \pm 2.0$ & $34.4 \pm 20.1$ \\
\hline Ciyanja & $2.0 \pm 0.0$ & $13.8 \pm 0.7$ & $10.6 \pm 3.2$ & $7.5 \pm 2.3$ & $6.1 \pm 2.3$ & $112.5 \pm 18.4$ & $3.3 \pm 2.4$ & $5.6 \pm 2.4$ \\
\hline Ibambiro & $2.9 \pm 0.3$ & $20.1 \pm 3.6$ & $12.2 \pm 3.2$ & $7.6 \pm 1.3$ & $5.9 \pm 1.2$ & $114.0 \pm 12.6$ & $3.4 \pm 1.2$ & $49.0 \pm 20.7$ \\
\hline Ikambi & $3.0 \pm 0.0$ & $22.3 \pm 5.0$ & $16.9 \pm 3.5$ & $9.3 \pm 2.4$ & $8.0 \pm 2.3$ & $79.5 \pm 20.1$ & $5.0 \pm 2.5$ & $66.2 \pm 28.4$ \\
\hline Kamagema & $2.4 \pm 0.5$ & $16.0 \pm 3.2$ & $12.8 \pm 4.4$ & $8.4 \pm 2.5$ & $7.2 \pm 2.5$ & $96.0 \pm 21.4$ & $5.2 \pm 2.3$ & $17.4 \pm 13.9$ \\
\hline Kasali & $2.8 \pm 0.4$ & $16.8 \pm 3.2$ & $13.8 \pm 3.5$ & $9.7 \pm 2.1$ & $7.5 \pm 2.9$ & $64.5 \pm 10.1$ & $5.3 \pm 2.3$ & $32.0 \pm 16.6$ \\
\hline Kazaroho & $3.0 \pm 0.0$ & $20.3 \pm 3.9$ & $11.8 \pm 4.2$ & $7.5 \pm 2.8$ & $6.4 \pm 2.5$ & $111.0 \pm 14.5$ & $3.5 \pm 1.3$ & $57.1 \pm 13.2$ \\
\hline Luzira & $2.1 \pm 0.3$ & $19.5 \pm 7.2$ & $19.2 \pm 2.5$ & $7.9 \pm 1.5$ & $7.0 \pm 1.4$ & $81.0 \pm 14.5$ & $4.3 \pm 1.4$ & $10.1 \pm 9.0$ \\
\hline Mashululwe & $2.8 \pm 0.4$ & $17.3 \pm 6.5$ & $13.6 \pm 4.3$ & $8.4 \pm 1.8$ & $8.0 \pm 1.8$ & $93.0 \pm 9.4$ & $6.5 \pm 4.3$ & $18.2 \pm 16.0$ \\
\hline Mulamba & $2.5 \pm 0.5$ & $16.5 \pm 5.3$ & $10.9 \pm 1.7$ & $8.6 \pm 2.3$ & $8.0 \pm 2.1$ & $99.0 \pm 14.5$ & $4.8 \pm 2.7$ & $26.9 \pm 27.5$ \\
\hline Mulengeza & $3.0 \pm 0.0$ & $18.1 \pm 6.0$ & $16.8 \pm 3.5$ & $9.5 \pm 2.8$ & $8.7 \pm 2.3$ & $87.0 \pm 9.4$ & $5.2 \pm 4.2$ & $48.5 \pm 30.2$ \\
\hline Mushenyi & $3.0 \pm 0.0$ & $19.8 \pm 8.4$ & $15.6 \pm 4.1$ & $9.2 \pm 2.8$ & $8.3 \pm 2.3$ & $96.0 \pm 12.6$ & $5.5 \pm 2.1$ & $39.2 \pm 25.4$ \\
\hline Nkungu & $2.6 \pm 0.5$ & $15.4 \pm 4.4$ & $13.8 \pm 3.5$ & $9.2 \pm 2.3$ & $8.5 \pm 2.8$ & $99.0 \pm 14.5$ & $4.9 \pm 2.0$ & $39.0 \pm 9.9$ \\
\hline Quartier C & $3.1 \pm 0.3$ & $16.1 \pm 2.7$ & $17.6 \pm 3.2$ & $9.5 \pm 1.8$ & $8.4 \pm 1.6$ & $78.0 \pm 21.0$ & $5.6 \pm 3.0$ & $9.9 \pm 11.3$ \\
\hline Total & $2.8 \pm 0.5$ & $18.8 \pm 6.1$ & $14.7 \pm 4.2$ & $8.8 \pm 2.3$ & $7.9 \pm 2.3$ & $88.2 \pm 21.7$ & $5.2 \pm 2.8$ & $34.1 \pm 25.2$ \\
\hline $\begin{array}{c}\text { Test Stat. Value } \\
\text { (F) }\end{array}$ & $8.689^{* * *}$ & $3.258^{\star * *}$ & $5.313^{\star * *}$ & $2.465^{\star * *}$ & $2.786^{\star * *}$ & $8.231^{\star * *}$ & $2.757^{\star \star \star}$ & $9.761^{* * *}$ \\
\hline
\end{tabular}

*** Significant at $\mathrm{P}<0.001$.

Nkungu $700.0 \pm 349.6 \mathrm{~g}$, hen in Ciyanja $1771.0 \pm 177.9 \mathrm{~g}$ and cock in Kamagema $2305.0 \pm 670.8 \mathrm{~g}$. There were high weights significant $(\mathrm{P}<0.001)$ differences in average across the sites of egg, chicken at weaning period, hen and cock.

\subsection{Parameters of Local Chicken Production}

The common chicken accommodations at Table 6 were chickens in the human house $29.4 \%$ followed by adobes kitchen and free-range $23.9 \%$ and house adobe and enclosure $7.8 \%$.

\subsection{Chicken Feeding Systems}

The feeding systems at Table 7 were dominated by the pure free-range $42.7 \%$, followed by free-range with grains given to the chickens $36.7 \%$, enclosure with concentrate $11.1 \%$, confined poultry where chickens were entirely nourished on concentrate $5.0 \%$. 
Table 4. Laying frequency and incidence of mortality per season.

\begin{tabular}{|c|c|c|c|c|c|}
\hline \multirow[t]{2}{*}{ Sites } & \multicolumn{3}{|c|}{ Laying frequency per week } & \multicolumn{2}{|c|}{ Mortality incidence per season } \\
\hline & Once each day & Once each 2 days & Once each three days & Wet season & Dry season \\
\hline Buholohombo & 6 & 4 & & 6 & 4 \\
\hline Cikonyi & 9 & 1 & & 8 & 2 \\
\hline Cimpunda & 3 & 7 & & 8 & 2 \\
\hline Cirhagabwa & 10 & & & 6 & 1 \\
\hline Ciriri & 9 & 1 & & 8 & 2 \\
\hline Ciyanja & 4 & 6 & & 6 & \\
\hline Ibambiro & 1 & 9 & & 7 & 3 \\
\hline Ikambi & 6 & 4 & & 4 & 4 \\
\hline Kamagema & 6 & 3 & 1 & 6 & 4 \\
\hline Kasali & 7 & 3 & & 7 & \\
\hline Kazaroho & 2 & 8 & & 9 & 1 \\
\hline Luzira & 2 & 8 & & 7 & 3 \\
\hline Mashululwe & 9 & 1 & & 4 & 6 \\
\hline Mulamba & 6 & 4 & & 4 & \\
\hline Mulengeza & 6 & 4 & & 5 & 5 \\
\hline Mushenyi & 8 & 2 & & 5 & 5 \\
\hline Nkungu & 9 & 1 & & 10 & \\
\hline Quartier C & 7 & 3 & & 1 & 9 \\
\hline Total & $110(61.1)$ & $69(38.3)$ & $1(0.6)$ & $111(68.5)$ & $51(3.5)$ \\
\hline
\end{tabular}

Values in parentheses are percentages.

Table 5. Weights of chickens and egg (g).

\begin{tabular}{|c|c|c|c|c|}
\hline Sites & Egg & Chicks weaning & Hen & Cock \\
\hline Buholohombo & $40.0 \pm 3.9$ & $392.8 \pm 124.9$ & $1271.9 \pm 152.9$ & $1683.1 \pm 327.6$ \\
\hline Cikonyi & $42.2 \pm 6.7$ & & $1146.9 \pm 131.8$ & $993.5 \pm 42.1$ \\
\hline Cimpunda & $45.4 \pm 1.0$ & $269.0 \pm 27.9$ & $1050.0 \pm 119.9$ & $1583.0 \pm 288.6$ \\
\hline Cirhagabwa & $44.2 \pm 6.3$ & $297.8 \pm 286.0$ & $1146.6 \pm 249.0$ & $1035.6 \pm 111.6$ \\
\hline Ciriri & $41.2 \pm 4.8$ & $538.9 \pm 153.7$ & $1540.0 \pm 263.3$ & $1740.0 \pm 275.7$ \\
\hline Ciyanja & $37.5 \pm 2.8$ & $566.2 \pm 91.6$ & $1771.0 \pm 177.9$ & $1864.0 \pm 215.3$ \\
\hline Ibambiro & $41.9 \pm 4.3$ & $340.9 \pm 75.1$ & $830.5 \pm 113.1$ & $904.2 \pm 190.5$ \\
\hline Ikambi & $43.7 \pm 2.9$ & $359.0 \pm 69.1$ & $1357.3 \pm 227.0$ & $1685.2 \pm 150.1$ \\
\hline Kamagema & $43.6 \pm 3.6$ & $534.1 \pm 160.3$ & $1731.0 \pm 341.8$ & $2305.0 \pm 670.8$ \\
\hline Kasali & $45.9 \pm 0.6$ & $298.9 \pm 15.7$ & $1177.5 \pm 147.8$ & $1773.0 \pm 143.8$ \\
\hline Kazaroho & $44.3 \pm 3.9$ & $223.3 \pm 2.9$ & $1147.7 \pm 322.6$ & $1866.6 \pm 672.0$ \\
\hline Luzira & $40.3 \pm 4.7$ & $590.5 \pm 350.2$ & $1257.8 \pm 255.4$ & $1748.6 \pm 299.4$ \\
\hline Mashululwe & $34.9 \pm 4.0$ & $470.6 \pm 67.5$ & $1256.0 \pm 201.7$ & $1430.0 \pm 316.4$ \\
\hline Mulamba & $41.3 \pm 2.1$ & $288.9 \pm 40.8$ & $1207.6 \pm 78.8$ & $1762.0 \pm 183.5$ \\
\hline Mulengeza & $45.7 \pm 3.1$ & $556.7 \pm 201.6$ & $1307.6 \pm 188.6$ & $1795.2 \pm 283.0$ \\
\hline Mushenyi & $46.6 \pm 5.2$ & $580,8 \pm 158.2$ & $1232.3 \pm 169.0$ & $1775.2 \pm 222.9$ \\
\hline Nkungu & $38.3 \pm 6.5$ & $700.0 \pm 349.6$ & $1380.0 \pm 253.0$ & $1690.0 \pm 303.5$ \\
\hline Quartier C & $42.5 \pm 4.7$ & $637.5 \pm 7.8$ & $1471.0 \pm 262.1$ & $1197.5 \pm 872.4$ \\
\hline Total & $42.3 \pm 5.1$ & $442.4 \pm 205.3$ & $1265.5 \pm 282.1$ & $1571.4 \pm 483.0$ \\
\hline Test Stat. Value (F) & $4.839^{* * *}$ & $7.025^{\star * *}$ & $8.685^{\star * *}$ & $8.353^{\star * *}$ \\
\hline
\end{tabular}

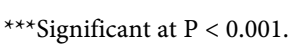


Table 6. Chicken accommodations.

\begin{tabular}{|c|c|c|c|c|c|c|c|c|c|c|c|c|c|c|c|c|c|c|c|}
\hline \multirow{2}{*}{ Chicken houses } & \multicolumn{18}{|c|}{ Sites } & \multirow[t]{2}{*}{ Total } \\
\hline & $A^{*}$ & B & C & $\mathrm{D}$ & $\mathrm{E}$ & F & G & $\mathrm{H}$ & I & $\mathrm{J}$ & $\mathrm{K}$ & $\mathrm{L}$ & $\mathbf{M}$ & $\mathbf{N}$ & $\mathrm{O}$ & $\mathbf{P}$ & Q & $\mathbf{R}$ & \\
\hline $\begin{array}{c}\text { Chickens accommodated in human } \\
\text { house }\end{array}$ & 2 & & 3 & 10 & 2 & 2 & 5 & & 3 & 8 & 4 & 1 & 3 & 1 & 3 & 2 & 2 & 1 & $53(29.4)$ \\
\hline Adobes kitchen and free-range & 5 & & & & 4 & 4 & 2 & & & & & 5 & 7 & 5 & 2 & 2 & 7 & & $43(23.9)$ \\
\hline House adobe and enclosure & 1 & & & & 1 & 1 & 2 & 3 & & & 1 & 3 & & 3 & 1 & & & & $16(8.9)$ \\
\hline Kitchen and free-range & & 1 & 1 & & 1 & 1 & & 2 & 2 & 1 & 1 & 1 & & 1 & 1 & & 1 & & $14(7.8)$ \\
\hline Planck's house and free-range & & 1 & & & 1 & 1 & & 2 & 2 & 1 & & & & & 1 & 3 & & 1 & $13(7.2)$ \\
\hline House adobe and enclosure & & 8 & & & & & & & & & 1 & & & & 1 & & & & $10(5.6)$ \\
\hline Planck's house and enclosure & & & & & 1 & 1 & & 2 & 2 & & 3 & & & & 1 & & & & $10(5.6)$ \\
\hline On a tree & & & 6 & & & & 1 & & & & & & & & & 2 & & & $9(5.0)$ \\
\hline Bricks house and enclosure & 1 & & & & & & & 1 & 1 & & & & & & & & & 2 & $5(2.8)$ \\
\hline Bricks kitchen and free-range & & & & & & & & & & & & & & & & & & 5 & $5(2.7)$ \\
\hline House on bricks and pavement & 1 & & & & & & & & & & & & & & & & & 1 & $2(1.1)$ \\
\hline Total & & & & & & & & & & & & & & & & & & & $180(100)$ \\
\hline
\end{tabular}

${ }^{*} \mathrm{~A}=$ Buholohombo, $\mathrm{B}=$ Cikonyi, $\mathrm{C}=$ Cimpunda, $\mathrm{D}=$ Cirhagabwa, $\mathrm{E}=$ Ciriri, $\mathrm{F}=$ Ciyanja, $\mathrm{G}=\mathrm{Ibambiro}, \mathrm{H}=\mathrm{Ikambi}, \mathrm{I}=\mathrm{Kasali}, \mathrm{J}=\mathrm{Kazaroho}, \mathrm{K}=\mathrm{Luzira}$, $\mathrm{L}=$ Mashululwe, $\mathrm{M}=$ Mulamba, $\mathrm{N}=$ Mulengeza, $\mathrm{O}=$ Kamagema, $\mathrm{P}=$ Mushenyi, $\mathrm{Q}=$ Nkungu and $\mathrm{R}=$ Quartier $\mathrm{C}$, Values in parentheses are percentages.

Table 7. Local chicken feeds.

\begin{tabular}{|c|c|c|c|c|c|c|c|c|c|c|c|c|c|c|c|c|c|c|c|}
\hline \multirow{2}{*}{ Feeding systems } & \multicolumn{18}{|c|}{ Sites } & \multirow[b]{2}{*}{ Total } \\
\hline & $A^{*}$ & $\mathrm{~B}$ & $\mathrm{C}$ & $\mathrm{D}$ & $\mathrm{E}$ & $\mathrm{F}$ & G & $\mathrm{H}$ & I & $\mathrm{J}$ & $\mathrm{K}$ & $\mathrm{L}$ & M & $\mathrm{N}$ & $\mathrm{O}$ & $\mathrm{P}$ & Q & $\mathrm{R}$ & \\
\hline Pure free-range & 9 & & 2 & 1 & 6 & 7 & 10 & 9 & 3 & 2 & 1 & 3 & 6 & 7 & 4 & 6 & 9 & 3 & $85(42.7)$ \\
\hline Free-range with grains & & 9 & 2 & 9 & & 2 & & & 2 & 7 & 4 & 6 & & 3 & 6 & 4 & 1 & 5 & $66(36.7)$ \\
\hline Enclosure and concentrate & & 1 & 4 & & 3 & 1 & & 1 & 3 & 1 & 5 & & 1 & & & & & 1 & $20(11.1)$ \\
\hline Confined poultry and concentrate & 1 & & 2 & & 1 & & & & 2 & & & 1 & & & & & & 1 & $9(5.0)$ \\
\hline Total & 10 & 10 & 10 & 10 & 10 & 10 & 10 & 10 & 10 & 10 & 10 & 10 & 10 & 10 & 10 & 10 & 10 & 10 & \\
\hline
\end{tabular}

${ }^{*} \mathrm{~A}=$ Buholohombo, $\mathrm{B}=$ Cikonyi, $\mathrm{C}=$ Cimpunda, $\mathrm{D}=$ Cirhagabwa, $\mathrm{E}=$ Ciriri, $\mathrm{F}=$ Ciyanja, $\mathrm{G}=$ Ibambiro, $\mathrm{H}=\mathrm{Ikambi}, \mathrm{I}=$ Kasali, $\mathrm{J}=\mathrm{Kazaroho}, \mathrm{K}=\mathrm{Luzira}$, $\mathrm{L}=$ Mashululwe, $\mathrm{M}=$ Mulamba, $\mathrm{N}=$ Mulengeza, $\mathrm{O}=$ Kamagema, $\mathrm{P}=$ Mushenyi, $\mathrm{Q}=$ Nkungu and $\mathrm{R}=\mathrm{Quartier} \mathrm{C}$, Values in parentheses are percentages.

With regard on Table 8, extension services didn't work normally; $91.7 \%$ of farmers were not assisted by them. Interviewees who didn't adhere to farmer's association represented at $98.5 \%$.

\subsection{Chicken Marketing}

Regarding Table 9, the averages price were respectively of egg $0.20 \pm 0.8 \$$, hen $6.5 \pm 1.8$ \$ and cock $9.7 \pm 1.9$ \$. There were high significant $(\mathrm{P}<0.001)$ differences in the average of all chicken's prices products across the sites. Then, prices are variously represented through the sites. Within the sites, hens from Ibambiro and Kasali were more profitable, it is the same to Buholohombo and Luzira for the cock and Ikambi the egg. Majority of chicken farmers had between 
Table 8. Extension services.

\begin{tabular}{|c|c|c|c|c|c|c|c|c|c|c|c|c|c|c|c|c|c|c|c|c|}
\hline & \multicolumn{20}{|c|}{ Sites } \\
\hline & Respoinse & $A^{*}$ & B & C & D & $\mathrm{E}$ & $\mathrm{F}$ & G & $\mathrm{H}$ & I & $\mathrm{J}$ & $\mathrm{K}$ & $\mathbf{L}$ & $\mathbf{M}$ & $\mathbf{N}$ & O & $\mathbf{P}$ & Q & $\mathbf{R}$ & Total \\
\hline \multirow{2}{*}{ Visit and support } & Yes & & & 2 & 1 & & 1 & & & & & 2 & & & 2 & 1 & & 3 & 3 & $15(8.3)$ \\
\hline & No & 10 & 10 & 8 & 9 & 10 & 9 & 10 & 10 & 10 & 10 & 8 & 10 & 10 & 8 & 9 & 10 & 7 & 7 & $165(91.7)$ \\
\hline \multirow{2}{*}{ Farmer's association } & Yes & & & & & & & & & & & 1 & & & & & 1 & & & $2(1.5)$ \\
\hline & No & 10 & 9 & 3 & 2 & 10 & 7 & 8 & 3 & 10 & 3 & 5 & 10 & 10 & 5 & 10 & 9 & 10 & 10 & $134(98.5)$ \\
\hline
\end{tabular}

${ }^{*} \mathrm{~A}=$ Buholohombo, $\mathrm{B}=$ Cikonyi, $\mathrm{C}=$ Cimpunda, $\mathrm{D}=$ Cirhagabwa, $\mathrm{E}=$ Ciriri, $\mathrm{F}=$ Ciyanja, $\mathrm{G}=$ Ibambiro, $\mathrm{H}=\mathrm{Ikambi}, \mathrm{I}=$ Kasali, $\mathrm{J}=\mathrm{Kazaroho}, \mathrm{K}=\mathrm{Luzira}$, $\mathrm{L}=$ Mashululwe, $\mathrm{M}=$ Mulamba, $\mathrm{N}=$ Mulengeza, $\mathrm{O}=$ Kamagema, $\mathrm{P}=$ Mushenyi, $\mathrm{Q}=$ Nkungu and $\mathrm{R}=$ Quartier $\mathrm{C}$, Values in parentheses are percentages.

Table 9. Chickens products prices and farmers income.

\begin{tabular}{|c|c|c|c|c|c|c|c|c|}
\hline \multirow[b]{2}{*}{ Sites } & \multicolumn{3}{|c|}{ Chicken's products prices (\$US) } & \multicolumn{5}{|c|}{ Monthly income (\$US) } \\
\hline & Hen & Cock & Eggs & 30 & $31-100$ & $101-200$ & $201-300$ & $301-400$ \\
\hline Buholohombo & $7.1 \pm 0.9$ & $11.8 \pm 0.9$ & $0.25 \pm 0.1$ & $1(4.7)$ & $3(7.9)$ & $4(6.4)$ & $1(3.4)$ & $1(20.0)$ \\
\hline Cikonyi & $5.8 \pm 1.5$ & $7.2 \pm 2.3$ & $0.15 \pm 0.0$ & & $3(7.9)$ & $4(6.4)$ & $2(6.7)$ & \\
\hline Cimpunda & $7.3 \pm 0.4$ & $10.7 \pm 0.9$ & $0.18 \pm 0.6$ & & $4(10.5)$ & $3(4.8)$ & $3(10.0)$ & \\
\hline Cirhagabwa & $5.1 \pm 1.1$ & $7.7 \pm 01.3$ & $0.25 \pm 0.0$ & $2(9.5)$ & & & & \\
\hline Ciriri & & & $0.16 \pm 0.5$ & $1(4.8)$ & $3(7.9)$ & $3(4.8)$ & $1(4.8)$ & $1(20.0)$ \\
\hline Ciyanja & $5.5 \pm 1.7$ & $8.8 \pm 0.6$ & $0.28 \pm 0.5$ & $5(23.8)$ & & & & \\
\hline Ibambiro & $9.6 \pm 0.3$ & $11.8 \pm 1.3$ & $0.28 \pm 0.1$ & & $2(5.3)$ & $5(7.9)$ & $2(6.7)$ & $1(20.0)$ \\
\hline Ikambi & & & $0.30 \pm 0.0$ & $1(4.8)$ & $2(5.3)$ & $3(4.8)$ & $1(3.3)$ & \\
\hline Kasali & $8.3 \pm 0.6$ & $9.2 \pm 1.4$ & $0.25 \pm 0.1$ & $2(9.5)$ & $5(13.1)$ & $2(3.2)$ & & $1(20.0)$ \\
\hline Kazaroho & $4.2 \pm 0.0$ & $9.8 \pm 0.5$ & $0.10 \pm 0.0$ & & & $5(7.9)$ & $3(10.0)$ & \\
\hline Luzira & $8.7 \pm 0.2$ & $11.4 \pm 1.4$ & $0.25 \pm 0.0$ & $1(4.8)$ & $2(5.3)$ & $6(9.5)$ & $1(3.3)$ & \\
\hline Mashululwe & $5.5 \pm 0.0$ & $9.9 \pm 0.5$ & $0.15 \pm 0.0$ & $2(9.5)$ & $1(2.6)$ & $4(6.3)$ & & \\
\hline Mulamba & 7.6 & 10.0 & $0.29 \pm 0.3$ & & $1(2.6)$ & $5(7.9)$ & $9(30.0)$ & \\
\hline Mulengeza & $6.6 \pm 0.8$ & $8.5 \pm 1.1$ & $0.25 \pm 0.0$ & & $3(7.9)$ & $5(7.9)$ & $1(3.3)$ & \\
\hline Kamagema & $7.9 \pm 1.7$ & $9.8 \pm 2.2$ & $0.25 \pm 0.0$ & $3(14.3)$ & $1(2.6)$ & $2(3.2)$ & $3(10.0)$ & $1(20.0)$ \\
\hline Mushenyi & $7.3 \pm 1.4$ & $9.5 \pm 2.2$ & $0.24 \pm 0.1$ & $2(9.5)$ & $3(7.9)$ & $3(4.8)$ & $2(6.7)$ & \\
\hline Nkungu & 5.8 & 7.8 & $0.15 \pm 0.5$ & $1(4.8)$ & $3(7.9)$ & $5(7.9)$ & $1(3.3)$ & \\
\hline Quartier C & $4.5 \pm 0.0$ & $9.7 \pm 0.9$ & $0.10 \pm 0.0$ & & $2(5.3)$ & $4(6.3)$ & & \\
\hline Total & $6.5 \pm 1.8$ & $9.7 \pm 1.9$ & $0.20 \pm 0.8$ & $21(13.3)$ & $38(24.1)$ & $63(39.9)$ & $30(19.0)$ & $6(3.8)$ \\
\hline Test Stat. Value (F) & $28.061^{\star * *}$ & $9.9676^{\star \star *}$ & $83.450^{\star * *}$ & & & & & \\
\hline
\end{tabular}

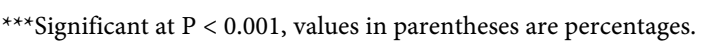

up 101 to 200 ser month $39.9 \%$. They are followed by those who had between 31 to $200 \$$ per month $24.1 \%$.

\section{Discussion}

\subsection{Livestock as an Asset to Push out Malnutrition and Poverty}

Farmers interviewed were yet young 39.3 years. Respondents' mean age was 40.9 
years in Sud-Kivu [9]. The gender issue on chicken farmers interviewed was dominated by $60.6 \%$ of males but in the same province, [10] observed that is was $54.3 \%$ of females who were interviewed on monogastric animal farmers. In Senegal poultry production is mainly managed by women and children [11]. Even if majority of chicken's farmers did at least the secondary school, $22.2 \%$ of illiterates are yet enough among farmers. According to the animal species, four sites had a good diversification. In TLU classification, swine got the first place followed by cattle. Cattle are symbol for peasants' social status and their possession reflects the wealth class that people belong to according to their self-perception [12]. Unhappily, cattle are not intended for regular consumption. The relationships of TLU with both education level and land size available point at multidimensional poverty restricting livestock husbandry [9]. This is why cattle are replacing slowly by other animal species such as swine in the region. The local chicken rate of mortality in Sud-Kivu is 34.1\%. The characteristics of these local chickens in Sud-Kivu are almost similar to those observed in Africa where $40 \%$ of the chicks die within the first $8-12$ weeks, hen lay in $3-4$ clutches of $10-15$ eggs each clutch a year and that is 30 to 60 eggs per year, hen weight 1170 to $1500 \mathrm{~g}$ as observed [13] [14]. To fight malnutrition and poverty, DRC government should promote chicken industry. As reproduction cycle of ruminants is long, a particular attention should be paid to promote the monogastrics animals. Non ruminants are prolific, robust animals have excellent meat quality; they can play an important role in fighting food insecurity [9]. We need a good program of local chickens characterization coupled with genetic molecular analysis in the entire Sud-Kivu province and why not in all the country before the selection begins.

\subsection{Regular Income Generation}

Majority of chickens traders had a revenue up 101 to $200 \$$ that is almost similar to Ethiopian where poultry farmer was between 91 to 150 \$ per month [15]. Chicken products prices are high in Bukavu town and his hinterland when in Tanzania, coq is cost $7 \$$ and hen $4.3 \$$ [13]. A poultry trader makes a monthly profit of 200 Birr (22 USD) [15]. The traditional system must be improved. It is possible in DRC to make benefit of 7 \$ monthly the first year and 107.3 \$ per month the second year on local chickens with at beginning of ten hens and one cock [16]. But also semi-intensive and intensive systems with improved chickens should be promoted to produce more food and income.

\section{Conclusion}

Survey described the local chickens systems in Bukavu and his hinterland located in Sud-Kivu DRC in terms of chicken production systems. The characteristics observed were on interviewees that male were more involved on local chickens than the female. Most of them were in secondary school and they were young yet. On local chickens production; the rate of mortality is high $34.1 \% \pm$ 
$25.2 \%$ mostly in wet season and chicks were more concerned. Local chickens lived on scavenging; accommodation was inadequate for the chickens. Weights of eggs, chicks at weaning period and hens were respectively $42.3 \pm 5.1 \mathrm{~g}, 442.4 \pm$ $205.3 \mathrm{~g}, 1265.5 \pm 282.1 \mathrm{~g}$ and cock $1571.4 \pm 483.0 \mathrm{~g}$. However, chickens products prices were high. Breeders and country deciders should continue with the local chicken characterization in the entire province. To start a good program of chicken industry in this province and as well as in all the country, selection of local chickens should be implemented on the basis of morphometric and genotypic.

\section{Acknowledgements}

Research was funded by the Korea-Africa Food Agriculture Cooperation Initiative.

\section{Conflicts of Interest}

The authors declare no conflicts of interest regarding the publication of this paper.

\section{References}

[1] SNSA (2011) Service national des statistiques agricoles RDC.

[2] Katunga, M.M.D., Muhigwa, B.J.B., Kashala, K.J.C., Kambuyi, M., Nyongombe, N., Maass, B.L. and Peters, M. (2014) Agro-Ecological Adaptation and Participatory Evaluation of Multipurpose Tree and Shrub Legumes in Mid Altitudes of Sud-Kivu, DRC. American Journal of Plant Sciences, 5, 2031-2039. https://doi.org/10.4236/ajps.2014.513218

[3] Ouma, E., Birachi, E., Pypers, P., Van Lauwe, B., Ekesa, B., Blomme, G., Chianu, J., Bouwmeester, H., Van Asten, P., Van Schagen, B., Nyagaya, M., Hakizimana, S., Bizimana, S., Gahigi, A., Ndayitegeye, O., Kantengwa, S., Lodi Lama, J., Katunga, M.M., Bishikwabo, K. and Vigheri, N.P. (2011) CIALCA Baselines Report. 142 p. http://www.cilaca.org

[4] Minagrdral (2013) Ministère de l'agriculture et du développement rural. Plan national d'investissement agricole PNIA 2013-2020 Ministère Agriculture et développement rural RDC. 210 p.

[5] SNSA (2013) Service national des statistiques agricoles R. D. Congo.

[6] Patrick, B.N., et al. (2019) Le système de production de la poule locale contraint son développement au Sud-Kivu, Est de la République Démocratique du Congo. Journal of Applied Biosciences, 135, 13821-13830. https://doi.org/10.4314/jab.v135i1.8

[7] Ghirotti, M. (1993) Rapid Appraisal: Benefiting from the Experiences and Perspectives of Livestock Breeders. World Animal Review, 77, 26-37. http://www.fao.org/docrep/V1650T/v1650T0d.htm

[8] LEAD (1999) Livestock, Environment and Development Initiative. Tropical Livestock Units (TLU). In: Livestock \& Environment Toolbox, LEAD/FAO, Rome. http://www.fao.org/ag/againfo/programmes/en/lead/toolbox/Mixed1/TLU.htm

[9] Maass, B.L., Katunga, M.M.D., Chiuri, W.L., Gassner, A. and Peters, M. (2012) Challenges and Opportunities for Smallholder Livestock Production in Post-Conflict South Kivu. Eastern DRC. Tropical Animal Health and Production, 44, 1221-1232.

https://doi.org/10.1007/s11250-011-0061-5 
[10] Katunga Dieudonné (2014) Evaluation des légumineuses fourragères dans un système d'élevage Editions universitaires européennes (08-07-2014). http://www.editions-ue.com

[11] El Hadji, T. (2014) Revues nationales de l'élevage Secteur avicole Sénégal de la division de la production et de la santé animales de la. FAO, No. 7, Rome.

[12] Katunga, M.M. (2004) Les systèmes agropastoraux du Bushi et du Buhavu à l'Est de la RDC face au défi de la malnutrition. Juin. CERPRU ISDR Bukavu RDC 89 p.

[13] Permin, A. (2009) Good Practices in Small Scale Poultry Production: A Manual for Trainers and Producers in East Africa. FAO, Rome.

[14] Sonaiya, E.B. and Swan, S.E. (2004) Production en Aviculture familiale. Organisation des Nations Unies pour l'Alimentation et l'Agriculture FAO, Rome, 140 p.

[15] Demeke, S. (2008) Poultry Sector Country Review of Ethiopia. FAO, Rome.

[16] Katunga, M.M.D. (2020) Manuel d'élevage des poules chez les petits éleveurs en République Démocratique du Congo Korea Rural Development Administration. Korea Food-Agriculture Cooperation Initiative and INERA, RDC RDA, 58 p. 